Regaña, C.B. , Piñero-Virué, R. P. \& Reyes-Rebollo, M.M. How Multimedia Support Collaborative Research: Best Practices

\title{
How Multimedia Support Collaborative Research: Best Practices
}

\author{
Cristóbal Ballesteros Regaña * \\ Universidad de Sevilla \\ cballesteros@us.es \\ Rocío P. Piñero-Virué \\ University of Seville \\ Miguel Ma Reyes-Rebollo \\ University of Seville \\ *Corresponding Author: cballesteros@us.es \\ Received : $\quad 02.07 .2019$ \\ Revision Requested: 06.09.2019 \\ Accepted : $\quad 09.20 .2019$
}

How to cite this paper: Regaña, C.B. , Piñero-Virué, R. P. \& Reyes-Rebollo, M.M. (2019). How Multimedia Support Collaborative Research: Best Practices. Research in Social Sciences and Technology, 4(2), 58-72.

\begin{abstract}
The purpose of this article is to evaluate the potential for online multimedia materials as alternative learning tools for the collaborative training of teachers in the design of researchbased teaching. A case study using a team of three trainee teachers was used to develop a process of initial training in the design of a constructivist and research-based teaching unit supported by online multimedia material. The results obtained from the research allowed some principles to be identified and basic recommendations and alternative lines of action to be given for designing online multimedia materials. Online multimedia materials can be alternative teaching tools that contribute, with other materials, to the development of constructivist, collaborative, and research-based strategies which help provide the necessary support for improving the initial and ongoing training of new teachers in the design of teaching from a research perspective.
\end{abstract}

Keywords: Multimedia design, Design of teaching, Collaborative teacher training, Teaching by research

\section{Introduction}

New information and communications technologies are the cornerstones that make online multimedia systems one of the most complete and capable educational tools for training teams of teachers. Within this framework, and equipped with a set of requirements which we aimed to define and test, we embarked on a research project to gain a better understanding of the potential for online multimedia material in initial training for teachers and in their subsequent professional development. It originated in the difficulties teaching teams face in addressing the design of constructivist and research-based teaching units in line with current trends in the Spanish curricular model (Ballesteros \& Román, 2016; Cabero \& Ballesteros, 2004). 
Regaña, C.B. , Piñero-Virué, R. P. \& Reyes-Rebollo, M.M. How Multimedia Support Collaborative Research: Best Practices

For this reason, based on issues related to the design and development of the curriculum, we set out to explore the extent to which online multimedia material, aimed specifically at training teaching staff in the design of units for teaching the sciences and environmental studies, could stimulate and facilitate the tasks involved in planning constructivist, research-based teaching units on specific issues pupils may encounter in their contact with the medium (Ballesteros \& Román, 2016).

Our study revolved around three fundamental questions. The first was how to make sure that teachers interested in constructivist and research-based learning could adequately address the task of designing teaching units of this nature (Austin, 2009). A particular problem we encountered here was how to determine the basic requirements for ensuring that teams of teachers could become involved in a collaborative way in designing their own teaching units (Kwan, Fox, Chan, \& Tsang, 2008).

However, given the difficulty of a large proportion of teachers' experience in undertaking these design tasks, we considered as a second fundamental question the problem of clarifying the barriers and difficulties they often face. We also looked at the issue of defining the characteristics of a strategy for collaborative professional development supported by online multimedia materials, and its potential for use in training (Cañal, Ballesteros, \& Merino, 2004).

The third research problem we addressed was connected to the previous one and focused very specifically on investigating the potential for online multimedia material in training teachers in processes for planning constructivist and research-based teaching units (Evans \& Gibbons, 2007). There is a clear gap and inadequacy of method regarding teaching design in a good proportion of initial and ongoing training courses for teachers, and there is also very little dedicated educational material to encourage training in this area or to facilitate design tasks (Cheng, Safont, Basu, \& Goebel, 2010). For this reason, we were interested in exploring the characteristics, potential, and limitations of a number of new materials for curricular development, which would not be limited to supplying information or resources for teaching traditional school topics. Instead, they would provide everything teaching teams needed to embark, with necessary support, on processes for reflective practice and planning, with defined study goals of interest to pupils. The materials would also allow them to make their own decisions about what to teach and how to teach it in an independent and informed way.

In this sense, we believed that online multimedia applications, along with a variety of other resources, could play a significant part in independent learning for teaching staff. We were equally aware, however, of the danger of overstating and generalizing the potential of these resources, bearing in mind the enormous diversity of contextual and motivational factors, as well as levels of professional development, etc., which could affect how each team of teachers might use them and how it might affect their usefulness in each case (Ballesteros, 2006; Lafer \& Tarman, 2019; Tarman, 2016). 
Regaña, C.B. , Piñero-Virué, R. P. \& Reyes-Rebollo, M.M. How Multimedia Support Collaborative Research: Best Practices

Our first goal, then, was to define the real difficulties facing teams of teachers who try to develop the design of their own teaching units in an attempt to go beyond blindly accepting and applying the concepts given in the textbooks. We were particularly interested in defining the difficulties implicit in these planning tasks without losing sight of the problems inherent in the real professional context in which these tasks are undertaken.

Once we had taken this initial step, we moved on to determining what kind of support online multimedia material could offer in addressing these difficulties and in facilitating the planning process, as well as contributing to the professional development of teachers in the use of this medium. This line of thought helped us to create a training strategy and determine its content using the online multimedia material we had produced (Cabero \& Ballesteros, 2004).

The next step in this process involved creating the multimedia material. As soon as it was ready, we put it into operation by arranging for a group of teachers in their initial stages of training to use it as an aid to planning a teaching unit on a specific study topic. This experimental process gave us the data we required for a preliminary assessment of the validity of our training strategy, the structure and content of the material, and the resources used in it. For this reason, the study was undertaken in four main phases:

1. Development of a theoretical/scientific framework for the research, and production of online multimedia support material for designing constructivist and research-based teaching units.

2. Setting the baseline data; determining the existing levels of professional development of the teachers taking part in the experiment.

3. Experiments using the online multimedia material.

4. Analysis of methods; conclusions.

Our ultimate aim was to support the initiatives of teaching staff interested in research-based learning in schools, experimentation with the curriculum, and the resulting improvement in school-based learning. With this in mind, we attempted to assess the extent to which the experimental online multimedia material could provide a prototype for enhancing and improving experience through an open process of experimentation in which teachers themselves were involved in pinpointing inadequacies and formulating proposals for change and improvement.

In terms of the above issues, we set the following research objectives:

1. To design and test a constructivist, research-based, and collaborative strategy for training teaching staff.

2. To design, produce, and test a set of online multimedia support materials for the design of research-based learning [M.A.D.E.I.].

3. To document the development of professional knowledge in a team of trainee teachers during the process of designing a constructivist teaching unit based on a strategy of learning through research. 
Regaña, C.B. , Piñero-Virué, R. P. \& Reyes-Rebollo, M.M. How Multimedia Support Collaborative Research: Best Practices

4. To identify the main learning opportunities and barriers encountered by teachers involved in designing this type of learning.

5. To give pointers, alternatives, and guidelines to help teachers interested in this form of learning to design and produce research-based teaching units.

6. To assess the usefulness and potential of online multimedia technology in general, and the M.A.D.E.I. material in particular, as a learning resource for training teaching staff in the design of research-based teaching units.

7. To discuss the implications of our study for the initial and ongoing training of teaching teams, and the development of new ways of experimenting with the curriculum.

\section{Characteristics of online multimedia material: Organizational and functional structure}

In describing the characteristics of the M.A.D.E.I. material (Ballesteros, 2008, 2016), we shall first give details of the structure for developing its content and layout, followed by details of the training strategy resulting from its organizational structure, which will inform its successful implementation.

\section{Organization}

To help design a constructivist and research-based teaching unit so that the actual educational resources were also in keeping with this focus, the M.A.D.E.I. material was organized around team-based study, reflection, and discussion on a set of specific questions to be addressed during a three-phase process:

1. Exploring and enhancing your professional knowledge:

a) What do we know and what do we need to know about constructivist and research-based teaching and units?

b) What do we know about plant nutrition?

2. Exploring pupils' ideas about plant nutrition:

c) How do primary pupils in general tend to conceptualize plant nutrition?

d) How do our pupils conceptualize plant nutrition?

e) What problems and obstacles are encountered in teaching plant nutrition in schools?

3. Planning a teaching unit:

f) What should the unit teach?

g) How should the teaching be organized?

The following structure was used to organize all this information within its own framework:

a) The interface was designed to ensure that the teaching team had access to all parts of the multimedia through the various levels of structuring and navigation.

b) The online multimedia used a navigation tree system, but within each module, teachers could move freely around the various sections and parts of the network. 
Regaña, C.B. , Piñero-Virué, R. P. \& Reyes-Rebollo, M.M. How Multimedia Support Collaborative Research: Best Practices

c) Although the modules were arranged in a constructivist and research-based teaching sequence, it was also possible, at the same time, for teachers to define their own way of using it according to their interests and their level of professional development.

d) Review tags in the upper left-hand corner of each screen pointed to the sections the teachers would be amending.

e) All screens were identified by their corresponding titles.

f) Intuitive icons played an important part in the design of the different elements of the interface and helped the user to navigate the multimedia.

g) To help motivate the user, the cursor changed form (an index finger on a hand pointed to active links, and a closed hand indicated the appearance of text with complementary information). Short help messages also came up on the symbols to explain their function.

h) The texts contained words or areas with two types of hyperlink: explanatory links to more information on content, and associative links that cross-referenced the different modules, sections, and parts of the online multimedia material in a meaningful way.

\section{Training strategy}

The functional structure for implementing the M.A.D.E.I. material emerged as a constructivist and research-based strategy for training teaching staff, with two main aspects:

a) It encouraged analysis, discussion, and progressive reformulation of professional knowledge as part of a research-based process led by the team of teachers involved in the design activities and who were supported and guided by it. In this way, the opening screen of each module of the multimedia contained sections called "Exploring our first ideas" and "What have we learned?" The first allowed them to make a preliminary analysis of their thoughts on the material that would be developed in each module. The second helped them to compare these initial ideas with the new knowledge they acquired at the end of the different training modules. b) It provided the necessary support for designing a teaching unit in keeping with a constructivist and research-based teaching strategy and, in terms of our particular study, it would contribute to the development of a specific study objective ("How do plants feed?") within primary education (6-12 years).

In this way, the M.A.D.E.I. material addressed the team of teachers on the one hand, and posed questions for them to study, reflect on, discuss, and make decisions about. On the other hand, it gave them direct access to information on these issues in a way that the teaching team considered most relevant and appropriate to its aims.

In short, our aim was to ensure that the online multimedia material offered the most appropriate type of support so that the unit produced by the teachers was genuinely innovative and could be put into practice by each member of the team. 
Regaña, C.B. , Piñero-Virué, R. P. \& Reyes-Rebollo, M.M. How Multimedia Support Collaborative Research: Best Practices

\section{Investigation methodology}

To overcome the problems and fulfill the objectives mentioned above, this research project used an initial training process for teaching staff which had been developed on the basis of online multimedia materials and specially designed for the purpose. To this end, a case study was proposed involving three students from the Faculty of Educational Sciences at the University of Seville (Spain), in which they had to use the online multimedia material (M.A.D.E.I.) to design a constructivist teaching unit based on a research-based teaching strategy.

The M.A.D.E.I. material was envisaged as support/guidance that would not only facilitate the design of teaching units on a specific theme (in our case, "plant nutrition") but would also contribute to the professional development of teachers interested in research-based teaching strategies. In terms of these factors, our proposed research had a twofold objective:

a) To understand how the professional knowledge of a team of trainee teachers developed while they were designing a constructivist teaching unit based on a research-based teaching strategy.

b) To assess the potential for using multimedia technology as an educational resource in developing processes for training school staff to design units using this type of teaching.

\section{The participants}

Given that the central objective of our research was to assess the potential of multimedia technology in general, and the M.A.D.E.I. material in particular, as an educational resource for the initial and ongoing training of teams of teachers in the design of research-based teaching units, the factors which influenced the sample used in the study were clearly influenced by this aim from the beginning.

For this reason, the teachers involved in the experimental team were chosen on the basis of a set of conditions which we considered desirable, and the criteria for selection were based on those suggested by Goetz and LeCompte (1988). They were as follows:

a) Willingness and personal availability to take part in the research project. The first condition was to choose trainee teachers who demonstrated genuine interest and who were available to take part in the different phases of the research. From the start, it was important that no teacher should be forced to take part in the study. On the contrary, after they had volunteered, they themselves would be the ones to decide their level of participation in the preliminary meetings, the general way in which they would be involved, and their commitment to decisions made at the meetings-in other words, everything to do with their availability in terms of the time and commitment expected of them during the research.

b) Enrollment in one of the specialization courses for the Educational Science qualification. In order to develop an effective preliminary training process with the M.A.D.E.I. material, we 
Regaña, C.B. , Piñero-Virué, R. P. \& Reyes-Rebollo, M.M. How Multimedia Support Collaborative Research: Best Practices

considered it important to opt for a condition which would cover all teachers in the experimental team; in our particular case, this was that they should still be studying to become future teaching professionals. From here, we would hone it down to students enrolled in one of the qualifications offered by the Faculty of Educational Sciences at the University of Seville (Spain). For this reason, the final team of teachers was composed of three students from the Faculty of Educational Sciences, two of whom were in their third year of studying primary education (6-12 years) and a third in the second year of studying nursery education (0-6 years). c) To have had different experiences of teaching methodologies over the course of their academic studies. The third criteria for selection for the sample group involved a preference that the teachers chosen for the case study should have had different experiences of teaching strategies throughout their education.

At the end of this process, we were able to form a final team of three trainee teachers with different backgrounds and formative experiences who also had little experience in taking part in training and research activities or projects, or in any type of course, seminar, conference, or activity of this nature.

\section{Instruments used}

A combination of four types of instruments were used: diaries (throughout the period where the research-based teaching unit was being designed) and group interviews with the team of participating teachers (at three key points in the experiment: at the beginning, halfway through, and at the end). Data was also collected from an analysis of the video recordings and from the documents and material produced using the online multimedia resources (notebook and teaching unit) in each of the joint design sessions and collaborative work developed over the course of the research.

In this sense, and following Schön (2008), the diaries became one of our main monitoring tools in considering a research model based on a continuous process of reflection and decisionmaking involving the teachers. The use of the diaries helped us to describe, analyze, and evaluate the sessions and the arguments that supported the dynamic of collaborative working developed during the second and third phases of the experiment. It brought us closer to the intended design strategy and gave us an idea of the thoughts and reflections emerging from the teaching team during the implementation of the various sessions. For each collaborative work session, the team of teachers kept a semi-structured diary with notes on two fundamental issues:

a) A description of the design strategy to show the steps followed by the teaching team in designing the teaching unit.

b) Reflections, analysis, and evaluation to show the various arguments that supported the design of the strategy. 
Regaña, C.B. , Piñero-Virué, R. P. \& Reyes-Rebollo, M.M. How Multimedia Support Collaborative Research: Best Practices

In considering the proposed research question and objectives, we decided to use a semistructured group interview framework with questions which were basically descriptive and structured. This allowed us to explore and detail both the professional knowledge the team of teachers was acquiring and the potential for using multimedia technology in training teaching teams to design research-based teaching units.

The interviews were conducted at three key points in the research: before, during, and at the end of the process of designing a teaching unit with the M.A.D.E.I. material. The semi-structural framework of the interviews allowed us to adapt and rework the questions we used, and also to incorporate new questions that arose in the course of the interviews.

The video recordings allowed us to record, observe, and analyze chronologically both the succession of events which were helping to form the design strategy and the reflective dynamic between the teachers while they were creating the teaching unit with the M.A.D.E.I. materials. The recordings were made throughout the development of the third phase of the research, and the camera remained fixed in a pan shot to capture the monitor screen and the sounds of the conversations and dialogues taking place at all times. This methodological procedure gave us a first-hand view of how they were using the online multimedia material, and gave us contextual information about the sequence and agenda followed by the teachers as they were designing the different curricular elements of the teaching unit.

Finally, the documents were reviewed in order to analyze the different items produced by the teaching team in each collaborative design and work session using the M.A.D.E.I. materials. In the course of the review, which took place during the development of the third and fourth research phases, we examined the following documents:

a) The notebooks the teachers were keeping to record their initial understanding, their conceptual constructs and re-evaluation of their ideas during the two M.A.D.E.I. sessions (Exploring our first ideas and What have we learned?) to give us an in-depth analysis of the different modules, as well as the participants' reflections, decision-making, questions, and issues. This document in particular was reviewed in both the third and fourth phases.

b) The teaching unit, which was constructivist and based on a strategy of teaching by research. It was created by the teaching team with the help of the M.A.D.E.I. material: How do plants feed? The unit was analyzed in the fourth and final phases.

\section{Data analysis}

Taking as our reference the theoretical framework of the study as well as the issues and objectives involved in the research, we defined a system for categorizing data with six dimensions, as follows: 
Regaña, C.B. , Piñero-Virué, R. P. \& Reyes-Rebollo, M.M. How Multimedia Support Collaborative Research: Best Practices

a) Teaching received throughout their education. Through this, we intended to detail the role played by the different elements of the curriculum in the teaching and learning processes the teachers had undergone from primary level (6-12 years) through secondary (12-16 years) and sixth form (16-18 years) to university level.

b) Current model for designing teaching units. We wanted to understand the team's initial ideas about their teaching model and teaching unit design.

c) Professional knowledge, which categorized the teachers' ideas on designing constructivist, research-based teaching units.

d) The potential for using the M.A.D.E.I. material in teaching. This assessed the possibilities for the use of online multimedia material in the actual development of a process for training teachers to design research-based teaching units.

e) General evaluation of the research, which included the team's own general assessments of the training process that was developed and the results obtained.

f) Suggestions, changes, and proposals for improvements to the research.

As soon as the system of data categorization had been set up, the first step involved using the various instruments to transform the set of information into data which could be more easily interpreted. In this way, and especially in the case of the interviews and video recordings, the information was transferred directly from its original medium onto computer, as the information from the remaining instruments had been collected digitally over the course of the research, through the diaries and documents in the notebook.

To analyze all the data obtained from the research, a content analysis technique was used involving a procedure in three main stages (Bardin, 2002):

1. Identification of units of information: coding. This first stage involved devising a general coding system for the data by identifying units of information, or "segments of content," as base units which would allow us to give an accurate account of the main characteristics of the content. In the specific context of our study, each unit of recorded data corresponded to an opinion, thought, commentary, question, issue or decision, i.e., any concrete evidence of the teachers' professional knowledge on any of the questions or issues they encountered during the research.

2. Categorization: Once the analytical units had been specified, they were classified and organized using the system of categorization designed for the research, so that all the information on each of the dimensions and categories in the system was arranged in a structured and systematic way. As a general principle, we tried to ensure that during the categorization process each unit of information corresponded exclusively to one dimension and category only, as "mutual exclusion" was the best way to ensure the effectiveness of the proposed system of categorization.

3. Interpretation or inference: Finally, the process of analyzing the data was completed in a third stage where we proceeded to interpret the different units of categorized information. In this 
Regaña, C.B. , Piñero-Virué, R. P. \& Reyes-Rebollo, M.M. How Multimedia Support Collaborative Research: Best Practices

way, taking as a reference the different dimensions and categories we had created in our system of categorization, we were able to integrate the various pieces of evidence from the teaching team in a meaningful way. This included their opinions, reflective thoughts, comments, queries, questions, and decision-making. In short, we were able to understand and analyze the development of the process they followed and the overall results obtained from the research as a whole.

Furthermore, during the final stage, the teaching team itself had a central role to play in the experience, specifically during the final interview, where they helped triangulate the data which had been collected, and the results of the four experimental phases, with the views of the teachers involved. This provided a safeguard against any bias in interpretation.

\section{Results}

It became increasingly clear that the teaching teams generally lacked training in organizing teaching processes. By way of offering an alternative to this situation, our research proposed to design and test a constructivist, research-based, and collaborative strategy for training that could inform the development of future processes of initial and ongoing training for teaching staff, and which would focus on teaching design through research. In this sense, the results obtained from the study provide evidence of the educational options on offer to support teachers through the whole process involved in designing and producing constructivist, research-based teaching units:

1. It contributes to the analysis and gradual reformulation of their professional knowledge in terms of the main learning elements on which the planning and design process is based.

a) Exploration and improvement of their professional knowledge in terms of the theme to be studied in the unit.

b) Exploration of the way primary pupils in general conceptualize the study theme and, in particular, the way pupils in their own classroom see it.

c) Planning the unit: What should the unit teach and how should the teaching be organized?

2. It creates a research process in which the teachers themselves are the main actors, taking part in the design tasks in an independent and collaborative way.

3. It adjusts to suit their design and learning abilities both as individuals and within the group.

4. It gives the learning support and guidance required to design this type of teaching unit, by:

a) taking account of constructivist approaches in order to exert a real influence on the curriculum in centers of learning;

b) balancing the commitments of teachers and pupils in the process of teaching and learning;

c) requiring teachers to be capable of guiding, adapting, and improving initial teaching proposals in the course of their development in order to adapt them to the ideas, experiences, and interests of the pupils themselves, and the obstacles they face;

d) enabling the development of real and meaningful learning based on the most immediate conceptual reality;

e) encouraging the involvement of pupils in developing all their learning activities; 
Regaña, C.B. , Piñero-Virué, R. P. \& Reyes-Rebollo, M.M. How Multimedia Support Collaborative Research: Best Practices

f) consolidating the role of pupils as active participants in building their own knowledge;

g) combining teaching and evaluation strategies into a single process to ensure continuous monitoring and adjustment throughout the whole training process. This will give it optimum coherence and consistency.

In terms of the potential for using multimedia technology in collaborative training of teaching staff in the design of teaching through research, as we have shown in the first part of this work, many of the problems currently observed in classrooms originated on the whole in the inadequacy of initial and ongoing training for teachers in designing teaching processes (Evans \& Gibbons, 2007; Tarman, 2012). This has been exacerbated by a lack of dedicated educational material which would provide the necessary support in overcoming these inadequacies (Cheng, Safont, Basu, \& Goebel, 2010). In this sense, the results of our research point to the following possible advantages of using multimedia technology:

1. It allows the design of flexible training environments that can adapt to different starting points and levels of professional development in teams of teachers interested in designing this type of teaching.

2. It takes into account their needs, initiatives, and professional concerns when they have to make decisions about which planning strategy to follow.

3. It gives the necessary support and guidance to reinforce their understanding of the main educational dimensions of the training process being developed.

4. It poses questions for them to study, reflect on, discuss and/or make decisions on, and at the same time provides access to information about these issues.

5. It opens up the possibilities for accessing and consulting any of its modules, sections, or parts. 6. It combines appearance and manageability in both the information and the various organizational resources it makes available (diaries, notebooks, design files, and individual products) so that a number of tasks can be developed at the same time on the same screen.

7. It combines the most useful aspects of conventional word processors for editing and manipulating written documents.

8. It adds key words and/or conceptual support diagrams to its various texts in order to give more information on meanings and basic relationships.

9. It saves space and teaching time.

10. It has excellent capacity for storing and organizing information in a number of forms, by combining it in a virtual space and making available the main sources of information which may be required to support the collaborative design of any research-based teaching unit.

11. It considerably reduces the time and effort required in design tasks for this type of teaching, bringing them in line with teachers' other professional work.

\section{Discussion and conclusions}

The characteristics of online multimedia educational material should be determined by the main organizational and functional factors which have emerged from the data we have presented: 
Regaña, C.B. , Piñero-Virué, R. P. \& Reyes-Rebollo, M.M. How Multimedia Support Collaborative Research: Best Practices

\section{About the first fundamental question:}

1.1. Respect and maintain the scientific quality and upgrading of the content that is developed. 1.2. Create open workspaces that allow the teachers to reflect and consult at all times, from their initial ideas to making decisions and/or the progress in the various collaborative work sessions.

1.3. Always take personal aspects of the teaching team into consideration so that they can move around independently to explore and consult the various sections and modules.

1.4. Facilitate procedures for obtaining feedback on the contents of the material and any information produced during design sessions.

\section{About the second fundamental question:}

2.1. Encourage professional motivation in the teaching teams in designing and determining the different curricular elements of the unit.

2.2. Make use of strategies to help the teachers exchange ideas and compare their initial concepts with their newly acquired learning. This will reinforce an active role for the teachers in building their new professional knowledge.

2.3. Allow each individual and the group as a whole to work at their own pace in learning and design so that the work is organized according to the group's own professional needs, knowledge, and concerns.

2.4. Facilitate collaborative and independent decision-making that provides the appropriate support and guidance required.

2.5. Make it possible to add new and complementary material that the teams decide to incorporate.

\section{About the third fundamental question:}

3.1. Adapt the language levels used in editing the contents to the background and needs of the teachers.

3.2. Throughout the design process, structure and organize the modules and sections so that the required information can be accessed easily.

3.3. Support navigation with pop-up conceptual diagrams that can provide an overview and show the main concepts and relationships for each issue on the same screen.

3.4. The graphics designed for the material (buttons, text, diagrams, and illustrations) need to contribute to the presentation and development of the contents so that they can be understood intuitively and aid navigation around the information. They also need to make it possible for the teachers to use a number of screens at the same time and therefore work on several tasks simultaneously.

3.5. Set up the collaborative work strategy so that all screens show the navigation paths and steps followed each time a user consults the material. 
In summary, we believe our study can make contributions in two main areas. On the one hand, it can help define a constructivist, research-based, and collaborative strategy for training teaching staff and help us overcome the main inadequacies in the initial and ongoing training of teachers with regard to the design of research-based teaching. On the other hand, the study can contribute to the design of new, specific, online multimedia teaching materials which will provide the necessary support in overcoming these deficiencies. 
Regaña, C.B. , Piñero-Virué, R. P. \& Reyes-Rebollo, M.M. How Multimedia Support Collaborative Research: Best Practices

\section{References}

Austin, K. A. (2009). Multimedia learning: Cognitive individual differences and display design techniques predict transfer learning with multimedia learning modules. Computers \& Education, 53(4), 1339-1354.

Ballesteros, C. (2006). E-herramientas de comunicación educativa. Comunicación y Pedagogía. Revista de Nuevas Tecnologías y Recursos Didácticos, 210, 55-63.

Ballesteros, C. (2008). M.A.D.E.I.: Material multimedia online de apoyo para el diseño de la enseñanza por investigación. Sevilla, España: Secretariado de Recursos Audiovisuales y Nuevas Tecnologías de la Universidad de Sevilla.

Ballesteros, C., \& Román, P. (2016). Experiencia de formación colaborativa para el diseño de unidades didácticas constructivistas e investigadoras. In D. Cobos, J. Gómez, y E. López (Coords.), La Educación Superior en el Siglo XXI: Nuevas Características Profesionales y Científicas (pp. 117-131). Puerto Rico: UMET Press, Universidad Metropolitana.

Bardin, L. (2002). El análisis de contenido. Madrid, España: Akal.

Cabero, J., \& Ballesteros, C. (2004). Posibilidades de los multimedia para la formación colaborativa del profesorado. Algunos principios y orientaciones didácticas para su diseño. Agenda Académica, 11 (1 y 2), 31-40.

Cañal, P., Ballesteros, C., \& Merino, J. (2004). Dificultades de los equipos de profesores en el diseño de unidades didácticas. Un material de apoyo multimedia. Investigación en la Escuela, 52, 57-67.

Cheng, I., Safont, L. V., Basu, A., \& Goebel, R. (2010). Multimedia in education: Adaptive learning and testing. New Jersey, USA: World Scientific Publishing.

Evans, C., \& Gibbons, N. J. (2007). The interactivity effect in multimedia learning. Computers \& Education, 49(4), 1147-1160.

Goetz, J. P., \& LeCompte, M. D. (1988). Etnografía y diseño cualitativo en investigación educativa. Madrid, España: Morata.

Kwan, R., Fox, R., Chan, F. T., \& Tsang, P. (2008). Enhancing learning through technology. New Jersey, USA: World Scientific Publishing.

Lafer, S., \& Tarman, B. (2019). Editorial 2019: (2)1, Special Issue. Journal of Culture and Values in Education, 2(1), i-v. Retrieved from http://cultureandvalues.org/index.php/JCV/article/view/34 
Ley 14/1970, de 4 de agosto, General de Educación (L.G.E.).

Ley Orgánica 8/1985, de 3 de julio, reguladora del Derecho a la Educación (L.O.D.E.).

Ley Orgánica 1/1990, de 3 de octubre de 1990, de Ordenación General del Sistema Educativo (L.O.G.S.E.).

Ley Orgánica 10/2002, de 23 de diciembre, de Calidad de la Educación (L.O.C.E.).

Ley Orgánica 2/2006, de 3 de mayo, de Educación (L.O.E.).

Schön, D. A. (2008). The formation of reflexive professionals: Towards a new design of the education and the learning in the professions. Barcelona, España: Paidós.

Tarman, B. (2012). Prospective teachers' beliefs and perceptions about teaching as a profession. Educational Sciences: Theory \& Practice, 12(3), 1-24.

Tarman, B. (2016). Innovation and Education. Research in Social Sciences and Technology, 1(1). Retrieved from http://ressat.org/index.php/ressat/article/view/3 